OPEN ACCESS

Edited by:

Guillermo Tellez,

University of Arkansas, United States

Reviewed by:

Victor Manuel Petrone-García, National Autonomous University of

Mexico, Mexico

Emanuel Gumina,

Vetanco S.A., Argentina

*Correspondence:

Yulong MaO

ylmaomail@126.com

Pengwei Zhao

zhaopengwei625@163.com

tThese authors have contributed equally to this work and share first

authorship

Specialty section: This article was submitted to Veterinary Infectious Diseases,

a section of the journal

Frontiers in Veterinary Science

Received: 01 January 2021 Accepted: 12 May 2021

Published: 22 June 2021

Citation:

Wang $B$, Ye $X$, Zhou $Y$, Zhao $P$ and Mao Y (2021) Glycyrrhizin Attenuates Salmonella Typhimurium-Induced

Tissue Injury, Inflammatory Response, and Intestinal Dysbiosis in C57BL/6 Mice. Front. Vet. Sci. 8:648698. doi: 10.3389/fvets.2021.648698

\section{Glycyrrhizin Attenuates Salmonella Typhimurium-Induced Tissue Injury, Inflammatory Response, and Intestinal Dysbiosis in C57BL/6 Mice}

\author{
Baikui Wang ${ }^{1 \dagger}$, Xiaolin Ye ${ }^{2 \dagger}$, Yuanhao Zhou ${ }^{1}$, Pengwei Zhao ${ }^{1 *}$ and Yulong Mao ${ }^{1 *}$ \\ ${ }^{1}$ Key Laboratory of Animal Molecular Nutrition of Education of Ministry, National Engineering Laboratory of Biological Feed \\ Safety and Pollution Prevention and Control, Key Laboratory of Animal Feed and Nutrition of Zhejiang Province, Institute of \\ Animal Nutrition and Feed Sciences, College of Animal Sciences, Zhejiang University, Hangzhou, China, ${ }^{2}$ Medical Faculty, \\ Heinrich-Heine University Düsseldorf, Düsseldorf, Germany
}

Salmonellae are one of the most important foodborne pathogens, which threaten the health of humans and animals severely. Glycyrrhizin (GL) has been proven to exhibit anti-inflammatory and tissue-protective properties. Here, we investigated the effects of GL on tissue injury, inflammatory response, and intestinal dysbiosis in Salmonella Typhimurium-infected mice. Results showed that GL or gentamicin (GM) significantly $(P<0.05)$ alleviated ST-induced splenomegaly indicated by the decreased spleen index, injury of liver and jejunum indicated by the decreased hepatocytic apoptosis, and the increased jejunal villous height. GL significantly $(P<0.05)$ increased secretion of inflammatory cytokines (IFN- $\gamma$, IL-12p70, IL-6, and IL-10) in spleen and IL-12p40 mRNA expression in liver. Meanwhile, GL or GM pre-infection treatments significantly $(P<0.05)$ decreased ST-induced pro-inflammatory cytokine (IFN- $\gamma$, TNF- $\alpha$, and IL-6) expression in both spleen and liver and increased $(P<0.05)$ anti-inflammatory cytokine $\mathrm{IL}-10$ secretion in spleen. Furthermore, GL or GM pre-infection treatment also regulates the diversities and compositions of intestinal microbiota and decreased the negative connection among the intestinal microbes in ST-infected mice. The above findings indicate that GL alleviates ST-induced splenomegaly, hepatocytic apoptosis, injury of jejunum and liver, inflammatory response of liver and spleen, and intestinal dysbacteriosis in mice.

Keywords: glycyrrhizin, Salmonella Typhimurium, tissue injury, inflammatory response, intestinal dysbiosis

\section{INTRODUCTION}

Salmonellae are facultative intracellular bacterial pathogens, which can invade and survive inside many cell types, such as epithelial cells, macrophages, microfold (M) cells, and dendritic cells $(1,2)$. As one of the most common foodborne pathogens, Salmonellae had strong pathogenicity, which colonizes, adheres to, and damages the intestinal epithelium by producing enterotoxins and then invade organs (such as liver, spleen, and kidney) by secreting invasive protein to induce organic swellings and inflammation $(3,4)$. Salmonellae-induced Salmonellosis poses a great threat to the health of food animals and humans. It is reported that Salmonellae-contaminated food-induced Salmonellosis is one of the major causes of diarrhea of humans globally (5), and the 
total economic loss caused by Salmonellosis is estimated to be over $\$ 3.5$ billion per year in the US (6). Salmonellae infection not only causes enteric diseases that compromise growth performance and overall health of animals and thereby lead to serious economic loss for food animal industry but also leads to serious Salmonellae-contaminated raw food animal products (meat and eggs) $(6,7)$. Salmonellae-contaminated poultry products (raw meat and eggs) are considered as the leading food sources for human Salmonellosis (8). Traditionally, in-feed antibiotics is the main strategy to prevent or control Salmonellae-induced enteric diseases in animal production (9). As growth promoters, antibiotics have been widely used in livestock production since the 1940's to improve growth performance and overall health of animals (10). However, with the increasing concerns about antimicrobial resistance (AMR) and foodborne antibiotic residues, many countries, including China, have banned the use of antibiotics in animal husbandry production (11-13). With the strict ban of the in-feed antibiotic growth promoters (AGPs), gastrointestinal infectious diseases of food animals and zoonotic pathogen contamination in animal products severely threaten the health of animals and humans $(11,14)$. Therefore, it is a priority to explore the proper alternatives to AGP under the post-AGP era (13). In recent years, many studies have been reported that some natural agents, such as natural plant extracts, probiotics, and prebiotics, are beneficial for improving growth performance and reducing morbidity and mortality of food animals, and are considered "Generally Recognized as Safe (GRAS)" alternatives to $\operatorname{AGP}(13,15-17)$.

Glycyrrhizin (GL), a triterpene glycoside that is extracted from licorice root, consists of one molecule $18-\beta$-glycyrrhetinic acid and two molecules of glucuronic acid and is the most important active ingredient of licorice root $(18,19)$. GL has been proven to exert a variety of pharmacological activities, such as anti-microbial, anti-inflammatory, anti-viral, anti-oxidative, anti-tumor, and hepatoprotective activities (18, 20-22). GL can activate specific and non-specific immune responses by enhancing phagocytosis and bactericidal activities of macrophage (18), inducing maturation of dendritic cells (DCs) and proliferation of $\mathrm{T}$ lymphocytes (23), augmenting natural killer (NK) cell activity (24), and inducing cytokine secretion (18). GL exhibits anti-inflammatory and tissueprotective properties by binding to high-mobility group box (HMGB1) to inhibit cytokine secretion activities (25). Many studies showed that as an alternative to antibiotics, GL had a beneficial effect in preventing or controlling multi-drugresistant pathogen infection $(26,27)$. As a potential substitute for AGP, licorice extract showed beneficial effects on the growth performance of broiler $(28,29)$. Our previous studies also found that GL exerts anti-Salmonella activities by inducing M1 polarization of murine bone marrow-derived macrophages (BMDMs) and maturation of murine bone marrow-derived dendritic cells (BMDCs) $(18,30)$. The present study was aimed to further investigate whether GL as a potential AGP substitute has protective effects against ST-induced tissue injury, inflammatory response, and intestinal dysbiosis in C57BL/6 mice.

\section{MATERIALS AND METHODS}

\section{Reagents}

GL was purchased from Sigma-Aldrich (purity $\geq 95.0 \%$, St. Louis, MO, USA). Gentamicin (GM) was purchased from SigmaAldrich (St. Louis, MO, USA). ELISA kits for IFN- $\gamma$, IL-12p70, TNF- $\alpha$, IL-6, and IL-10 were obtained from eBioscience (San Diego, CA, USA). Caspase-1 activity assay kit was purchased from Beyotime Institute of Biotechnology (Shanghai, China).

\section{Bacteria Preparation}

ST CMCC 50115 was generously provided by Dr. Weihuan Fang (Institute of Preventive Veterinary Medicine, Zhejiang University). ST was cultured at $37^{\circ} \mathrm{C}$ in Luria-Bertani broth overnight under aerobic conditions. The ST pellet was harvested by centrifugation at $4000 \times g$ for $15 \mathrm{~min}$ at $16^{\circ} \mathrm{C}$ and then washed three times with sterile phosphate-buffered saline $(\mathrm{pH}$ 7.2). Finally, the optical density method (SpectraMax M5, MD, USA) and spreading plate method were conducted to adjust the final ST.

\section{Animal Experimental Design}

Male C57BL/6 mice (6 weeks old, Slac Animal Inc., Shanghai, China) were raised in the Experimental Animal Center of Zhejiang University under light-controlled (12-h light/dark cycle) and temperature-controlled $\left(22 \pm 1^{\circ} \mathrm{C}\right)$ conditions and had free access to water and food. Fifty male C57BL/6 mice were randomly divided into five groups ( $n=10 /$ group): Control group, glycyrrhizin-treated group (GL), ST-infected group (ST), glycyrrhizin protective group (GL + ST), and gentamicin protective group (GM $+\mathrm{ST}$, as a positive protective group). Mice in the Control and ST groups were drinking sterile water. The mice in the GL, GL + ST, and GM + ST groups were drinking sterile water containing $0.4 \mathrm{mg} / \mathrm{ml} \mathrm{GL}$ ( $80 \mathrm{mg} / \mathrm{kg}$ weight) or $0.4 \mathrm{mg} / \mathrm{ml} \mathrm{GM}$ (80 mg/kg weight) every day, respectively (30). All mice were fed a basal diet and weighted every 3 days. After 3 weeks, mice were orally infected with $200 \mu \mathrm{l}$ of Salmonella Typhimurium $\left(2 \times 10^{9} \mathrm{CFU} / \mathrm{ml}\right)(31)$. Mice were euthanized at day 3 post-infection. Spleen was weighted, and liver, jejunum, and cecum were collected for further analysis. Spleen index $(n=$ 10/group) was calculated according to the formula: Spleen index $=$ spleen weight $(\mathrm{mg}) /$ body weight $(\mathrm{g})$.

\section{Hematoxylin and Eosin Staining and TUNEL Assay}

For photonic microscope observations, the liver and jejunal samples ( $n=5$ /group) of mice were fixed in $4 \%$ paraformaldehyde, embedded in paraffin, sliced, dehydrated with gradient concentrations of alcohol, and then stained with hematoxylin and eosin (H\&E). Images were captured, and the villus height was measured by an Olympus microsystem (Tokyo, Japan). The TUNEL assay was determined by using TUNEL Assay Kit (Abcam, Cambridge, United States) according to the manufacturer's instructions. Briefly, the paraffin-embedded liver sections were deparaffinized with xylene, hydrated with gradient concentrations of alcohol, and covered with proteinase K. Slides were incubated with terminal deoxynucleotidyl transferase 
TABLE 1 | Primer sequences used for qRT-PCR.

\begin{tabular}{|c|c|c|c|}
\hline Gene name & Primer sequence $\left(5^{\prime}-3^{\prime}\right)$ & $\begin{array}{l}\text { Product } \\
\text { size }\end{array}$ & Accession no. \\
\hline \multirow[t]{2}{*}{ TNF- $\alpha$} & F: CCCTCACACTCAGATCATCTTCT & 61 & NM_013693 \\
\hline & R: GCTACGACGTGGGCTACAG & & \\
\hline \multirow[t]{2}{*}{ IL-12 p40 } & F: СССАТТССТАСТТСТСССТСАА & 75 & NM_001303244 \\
\hline & R: ССTССTCTGTCTCCTTCATCTT & & \\
\hline \multirow[t]{2}{*}{ IL-6 } & F: TAGTCCTTCCTACCCCAATTTCC & 76 & NM_031168 \\
\hline & R: TTGGTCCTTAGCCACTCCTTC & & \\
\hline \multirow[t]{2}{*}{ IFN- $\gamma$} & F: TCAGCAACAACATAAGCGTCAT & 104 & NM_008337 \\
\hline & R: GACCTCAAACTTGGCAATACTCA & & \\
\hline \multirow[t]{2}{*}{ IL-10 } & F: GCTCTTACTGACTGGCATGAG & 105 & NM_010548 \\
\hline & R: CGCAGCTCTAGGAGCATGTG & & \\
\hline \multirow[t]{2}{*}{$\beta$-actin } & F: CGTTGACATCCGTAAAGACC & 281 & NM_007393 \\
\hline & R: AACAGTCCGCCTAGAAGCAC & & \\
\hline
\end{tabular}

TNF- $\alpha$, tumor necrosis factor alpha; IL, interleukin; IFN- $\gamma$, interferon gamma; $F$, forward; $R$, reverse.

and biotinylated nucleotides and then treated with salinesodium citrate buffer, $6 \%$ hydrogen peroxide, streptavidin-HRP conjugate, and DAB substrate solution. Finally, the slides were counterstained in hematoxylin solution. Images were captured using an Olympus microsystem (Tokyo, Japan) and the apoptotic cells were quantified using ImageJ analysis software (National Institute of Mental Health, Maryland, USA). Apoptosis index was calculated according to the formula: Apoptosis index (\%) = [the number of apoptotic cells/(the number of apoptotic cells + the number of intact cells)] $\times 100$.

\section{ELISA and Caspase-1 Activity Analyses}

The spleen and liver samples ( $n=5$ /group) were homogenized with ice-cold sterile saline solution $(1: 9, \mathrm{w} / \mathrm{v})$ and centrifuged at $4000 \times g$ for $20 \mathrm{~min}$ at $4{ }^{\circ} \mathrm{C}$. Then, the collected supernatant was used for ELISA and caspase-1 activity analyses. Levels of interferon- $\gamma$ (IFN- $\gamma$ ), interleukin-12 subunit p70 (IL12p70), tumor necrosis factor- $\alpha$ (TNF- $\alpha)$, IL-6, and IL-10 in the spleen homogenates were colorimetrically determined by enzyme-linked immunosorbent assay (ELISA) kits (eBioscience, San Diego, CA) according to the manufacturer's instructions. Caspase-1 activity in liver homogenates were determined by caspase-1 activity assay kit according to the manufacturer's instructions.

\section{RT-qPCR Analysis}

Total RNA ( $n=5$ /group) was extracted from spleen tissue using RNAiso Plus kit (TAKARA, Dalian, China). Reverse transcription was performed using the PrimeScript II 1st Strand cDNA Synthesis Kit (TAKARA) according to the manufacturer's recommendation. RT-qPCR was conducted using SYBR PremixEx TaqII (TAKARA) by the StepOne real-time PCR system (Applied Biosystems). All primer sequences for target genes (including TNF- $\alpha$, IL-12p40, IL-6, IFN- $\gamma$, and IL-10) are listed in Table 1. Fold changes were calculated after normalizing to the housekeeping gene $\beta$-actin using the $2^{-\Delta \Delta \mathrm{Ct}}$ method.

\section{Microbial Analysis}

The cecal bacterial genomic DNA ( $n=4$ /group) was extracted using the TIANamp Stool DNA Kit (Tiangen, Beijing, China) according to the manufacturer's instructions, and the quality of extracted DNA was checked by agarose gel electrophoresis and spectrophotometric analysis. The V3-V4 region of the $16 \mathrm{~S}$ rRNA gene was amplified using the primer pair $341 \mathrm{~F} / 805 \mathrm{R}$, and sequencing was performed on MiSeq platform (Illumina Inc., San Diego, CA, USA). Sequences were filtered and clustered into operational taxonomic unit (OTU) at $97 \%$ similarity by QIIME software (version 1.9.1). Bacterial OTU representative sequences were assigned to a taxonomic lineage by Ribosomal Database Project (RDP) classifier based on the SILVA database (SILVA 132 release).

Alpha diversity (observed OTUs and PD_whole_tree) and beta diversity were analyzed based on a subsample of a minimum number of sequences $(12,722)$ by QIIME software. Beta diversity was displayed by principal coordinates analysis (PCoA) using R software (https://www.r-project.org/). Analysis of similarities (ANOSIM), permutational multivariate analysis of variance (PERMANOVA), and multi response permutation procedure (MRPP) were calculated using "vegan" package of R software to determine significant differences in bacterial beta diversity among the five groups (based on the Bray-Curtis distance matrices) (32).

\section{Statistical Analysis}

Pearson correlation between phenotypic variables and the relative abundance of microbial communities (phylum level) were analyzed and visualized by the package "corrplot" of $\mathrm{R}$ software. The co-occurrence networks of microbial communities in different treatments were built based on significant correlations (Spearman's $R>0.6$ and FDR-adjusted $P$ $<0.05$ ) (33) and were visualized by Gephi ${ }^{\mathrm{TM}}$ software (https:// gephi.org/). The topological properties of the co-occurrence network were also calculated to describe the complex patterns of the interrelationships by Gephi software. Comparison of the intestinal bacteria among different treatments was analyzed and visualized by statistical analysis of taxonomic and functional profiles (STAMP) with a 95\% confidence interval. The rest of the data were evaluated by ANOVA, and the contrast of means was performed by Tukey's multiple range test, using SPSS ${ }^{\mathrm{TM}}$ software (SPSS Inc., Chicago, IL, USA), and the graphs were generated using Origin $8.5^{\mathrm{TM}}$ (OriginLab, Berkeley, CA, USA). The statistical significance was set at $P<0.05$.

\section{RESULTS}

\section{GL Attenuates ST-Induced Jejunum Injury}

$\mathrm{H} \& \mathrm{E}$ staining showed that compared with the Control group, GL treatment had little effect on villous height $(P>0.05)$ and jejunal structure, which exhibited integrated structure, ordered jejunal villi, and completed gland (Figure 1). However, compared with the Control group, ST infection significantly $(P<0.05)$ decreased the villous height of jejunum. Compared with the ST group, GL or GM significantly $(P<0.05)$ prevented jejunum injury indicated by the higher villous height (Figure 1). 


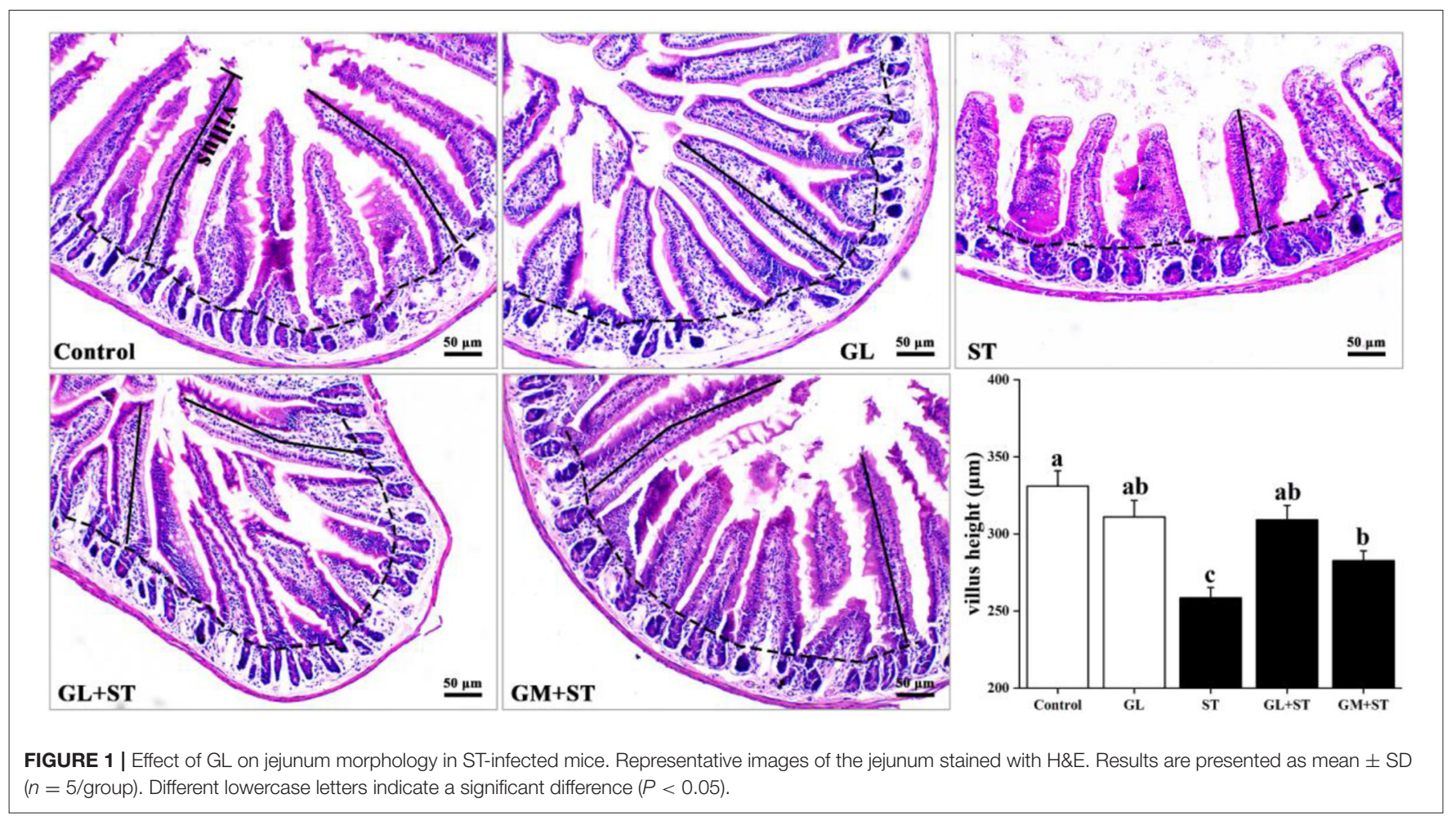
$(n=5 /$ group). Different lowercase letters indicate a significant difference $(P<0.05)$.

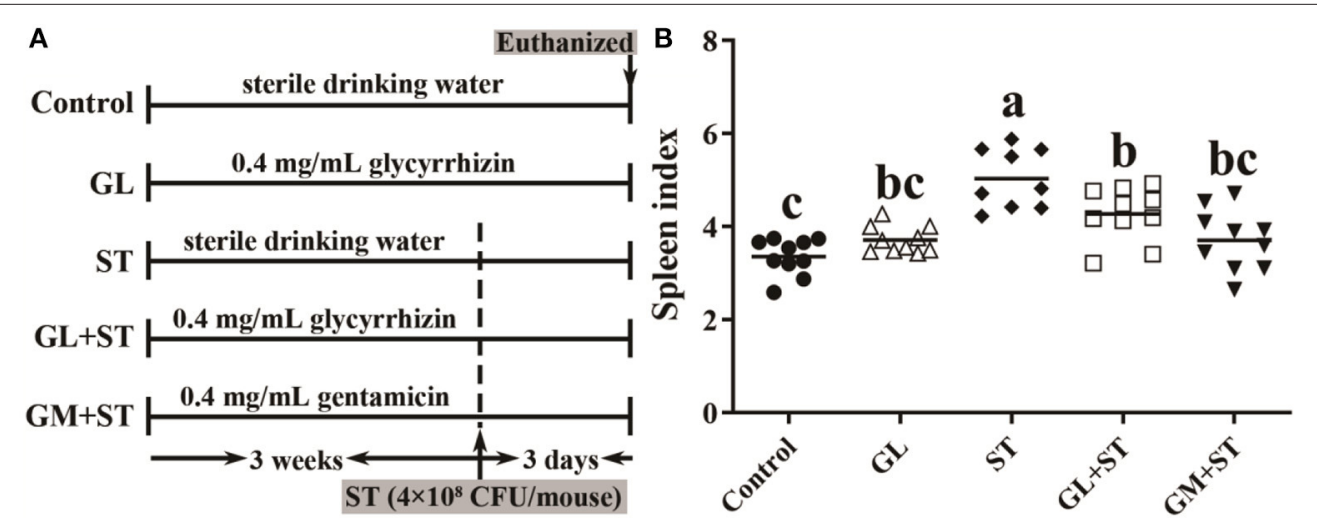

FIGURE 2 | GL alleviates ST-induced splenomegaly. (A) Graphical outline of the animal study. (B) Spleen index. Results are presented as mean \pm SD ( $n=10 / g r o u p)$. Different lowercase letters indicate a significant difference $(P<0.05)$.

\section{GL Alleviates ST-Induced Splenomegaly}

As shown in Figure 2, compared with the Control group, the spleen index of mice was significantly $(P<0.05)$ increased in the ST-infected group by $50.18 \%$. GL or GM pretreatments significantly $(P<0.05)$ attenuated ST-induced splenomegaly by 15.16 or $26.35 \%$, while there was no significant difference between the two pretreated groups $(P>0.05)$.

\section{GL Attenuates ST-Induced Liver Injury}

Compared with the Control group, GL treatment did not cause hepatocytic apoptosis exhibiting completed structure, hepatic lobule, and intact cell and unincreased apoptosis index $(P>0.05)$ (Figures 3A,B). However, ST infection caused hepatocytes with fragmented and pyknotic nuclei (Figure 3A, H\&E, black arrows indicted) and hepatocytic apoptosis (Figure 3A, TUNEL, cells labeled as brown) and significantly $(P<0.05)$ increased apoptosis index (Figure 3B), which could be significantly $(P<0.05)$ alleviated by GL or GM pretreatments (Figures 3A,B). Compared with the Control group, GL treatment had no effect on caspase1 activity $(P>0.05)$, while ST infection significantly $(P<0.05)$ increased the activity of caspase-1, which could be significantly $(P<0.05)$ reversed by GL or GM pretreatments (Figure 3C). 

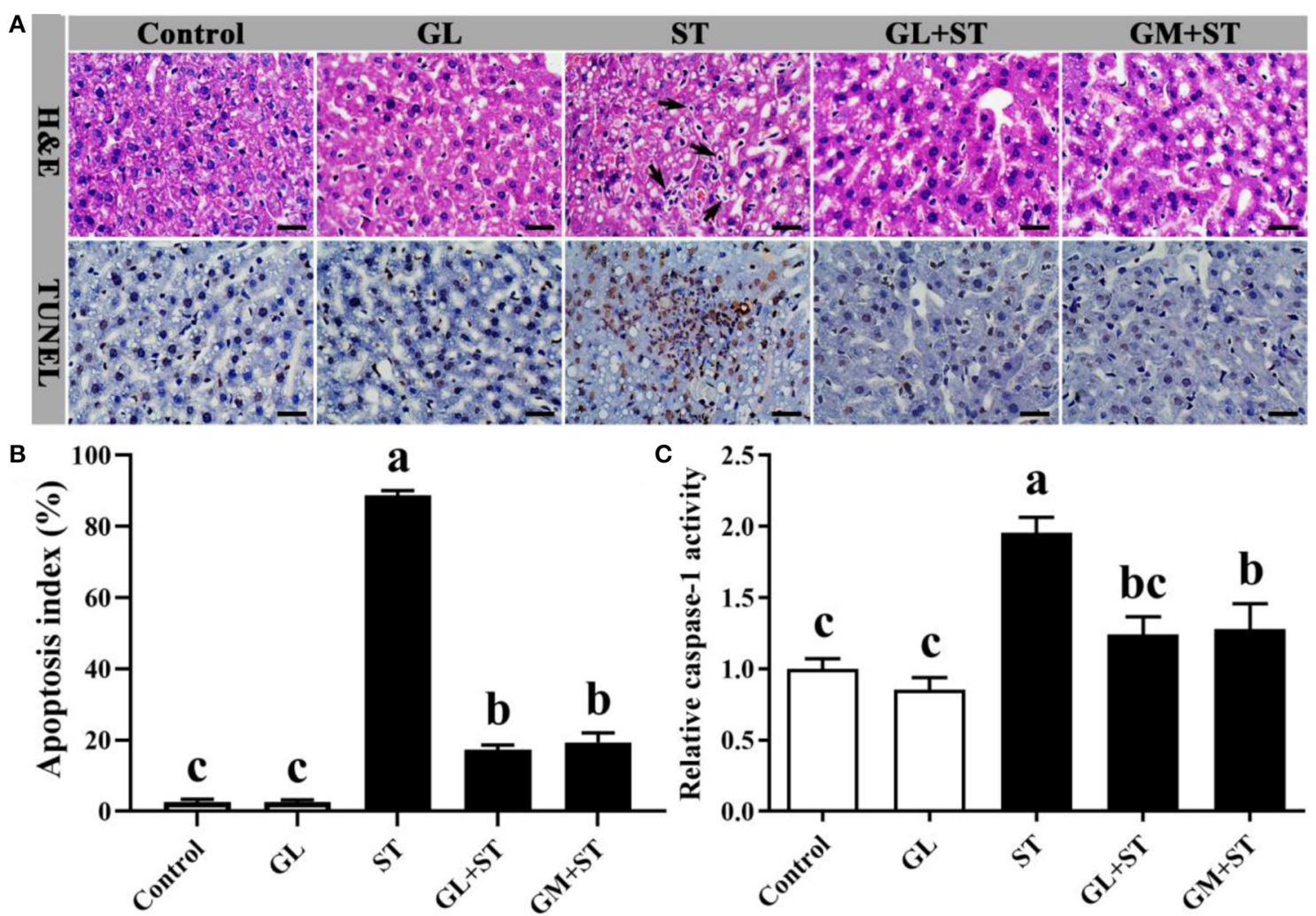

FIGURE 3 | Effect of GL on liver morphology and hepatocytic apoptosis in ST-infected mice. (A) H\&E staining and TUNEL assay (scale bar, 50 m). H\&E: Black arrow indicates apoptotic cells. TUNEL assay: Apoptotic cells were labeled as brown, and intact cells were labeled as blue. (B) Apoptosis index was calculated according to the formula: Apoptosis index (\%) = [the number of apoptotic cells/(the number of apoptotic cells + the number of intact cells)] $\times 100$. (C) Caspase-1 activity in liver was determined by caspase- 1 activity assay kit. Results are presented as mean \pm SD ( $n=5 /$ group). Different lowercase letters indicate a significant difference $(P<0.05)$.

\section{GL Alleviates ST-Induced Spleen and Liver Inflammation}

As shown in Figure 4, compared with the Control group, GL treatment significantly $(P<0.05)$ increased the secretions of IFN- $\gamma$, IL-12p70, IL-6, and IL-10 in spleen, whereas, it had no $(P>0.05)$ effect on the protein level of TNF- $\alpha$. ST infection significantly $(P<0.05)$ increased the secretion of proinflammatory cytokines (IFN- $\gamma$, IL-12p70, TNF- $\alpha$, and IL-6) and anti-inflammatory cytokine (IL-10) in spleen. As expected, GL or GM pretreatments effectively $(P<0.05)$ decreased the increased pro-inflammatory cytokines (IFN- $\gamma$, TNF- $\alpha$, and IL-6) induced by $\mathrm{ST}$ infection, whereas, they increased anti-inflammatory cytokine IL-10 secretion $(P<0.05)$.

Furthermore, the mRNA expression of cytokines in liver showed that compared with the Control group, GL treatment significantly $(P<0.05)$ upregulated the IL-12p40 mRNA expression, whereas, it had no significant $(P>0.05)$ effect on the expression of IFN- $\gamma$, TNF- $\alpha$, IL-6, and IL-10 (Figure 5). ST infection significantly $(P<0.05)$ increased the mRNA expression of pro-inflammatory cytokines (IFN- $\gamma$, IL-12p40, TNF- $\alpha$, and IL6) and anti-inflammatory cytokine (IL-10). Similarly, GL or GM pretreatments also significantly $(P<0.05)$ decreased the mRNA expression of IFN- $\gamma$, TNF- $\alpha$, and IL-6 (Figure 5).

\section{GL Modulates ST-Induced Intestinal Dysbiosis}

Alpha diversity analysis showed that compared with the Control group, GL treatment significantly $(P<0.05)$ increased the alpha diversities (including observed OTUs, PD_whole_tree index, Chaol, and Ace) of the intestinal microbiota, while ST infection had no $(P>0.05)$ effect on the alpha diversities (Figure 6). Additionally, the alpha diversity indices of the microbial communities in the GL + ST and $\mathrm{GM}+\mathrm{ST}$ treatment were significantly $(P<0.05)$ lower than those of the ST group. PCoA of microbial communities based on Bray-Curtis distance suggested variation of the bacterial community structure with treatments (Figure 7). Significant differences in beta diversity among treatments were further confirmed by ANOSIM, PERMANOVA, and MRPP analysis (Table 2), except for that between the Control and GL treatments.

The differences of the intestinal bacterial compositions among groups were also analyzed (Figure 8). The results showed that GL treatment significantly $(P<0.05)$ increased the relative abundance of Oscillibacter, Millionella, and Bilophila, whereas, it decreased $(P<0.05)$ the relative abundance of Verrucomicrobiales, Family XIII AD3011 

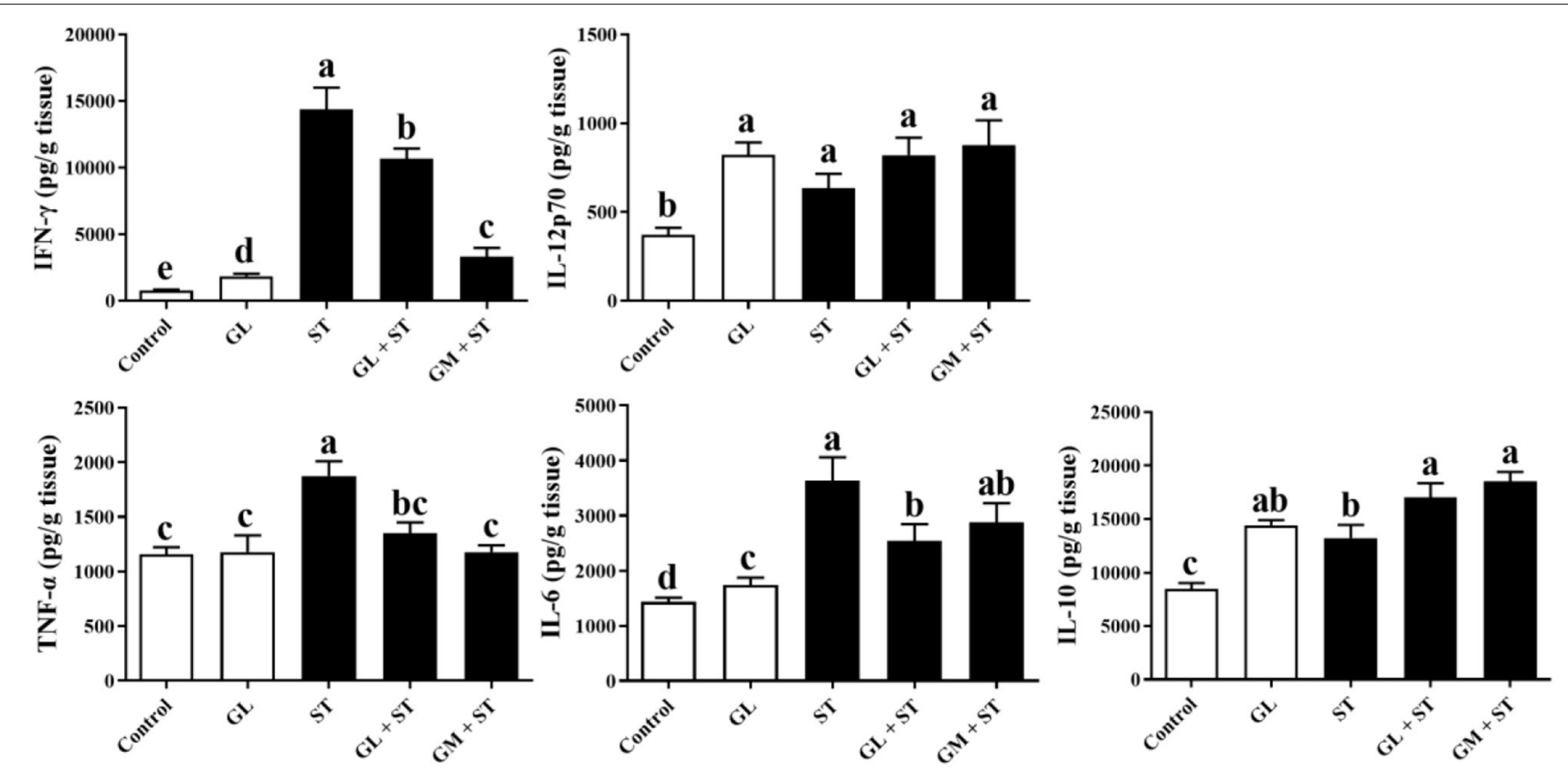

FIGURE 4 | Effect of GL on splenic cytokine production in ST-infected mice. The levels of pro-inflammatory cytokine (IFN- $\gamma$, IL-12p70, TNF- $\alpha$, and IL-6) and anti-inflammatory cytokine (IL-10) in spleen were determined by ELISA kit. Results are presented as mean \pm SD ( $n=5 / g r o u p)$. Different lowercase letters indicate a significant difference $(P<0.05)$.

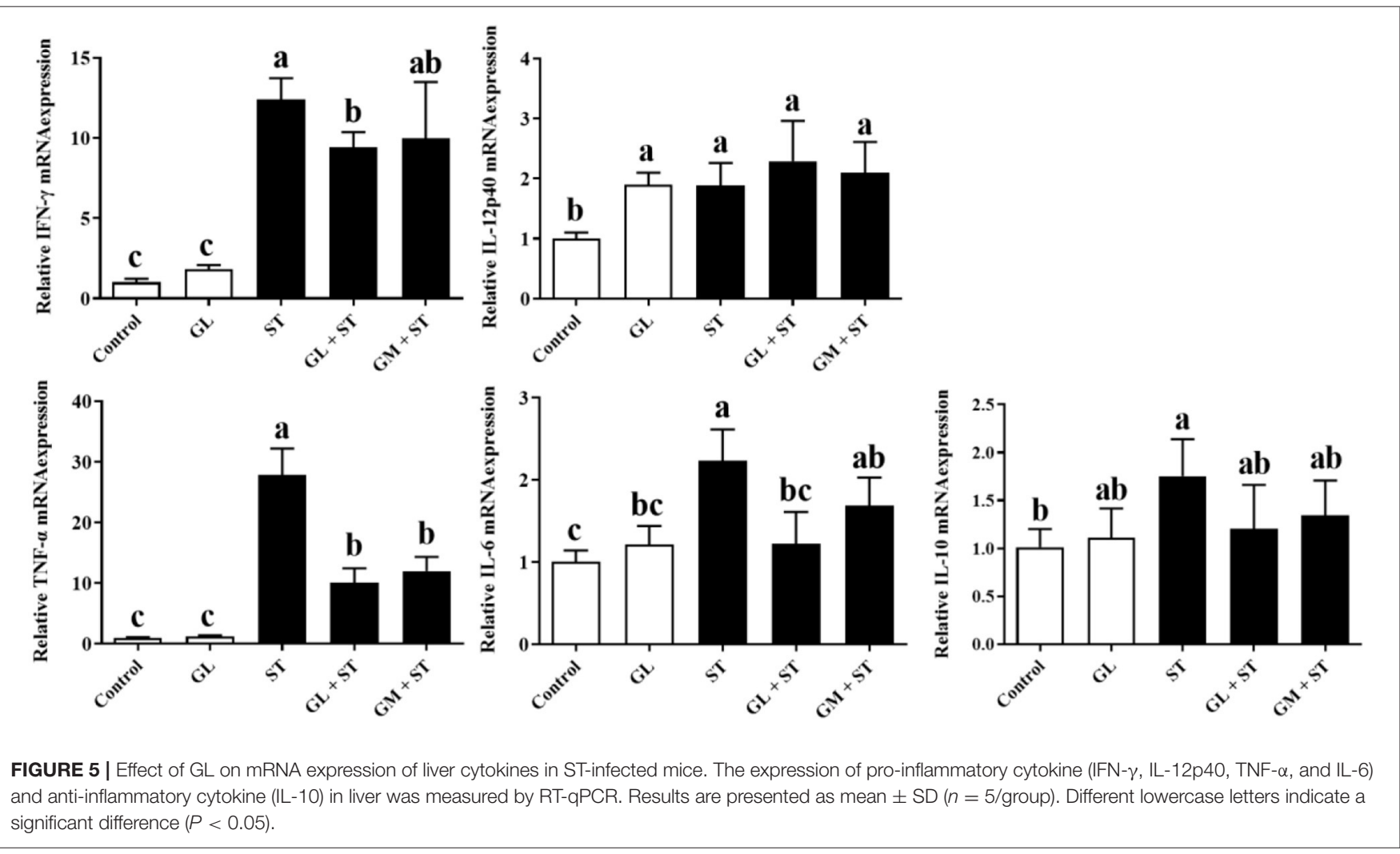




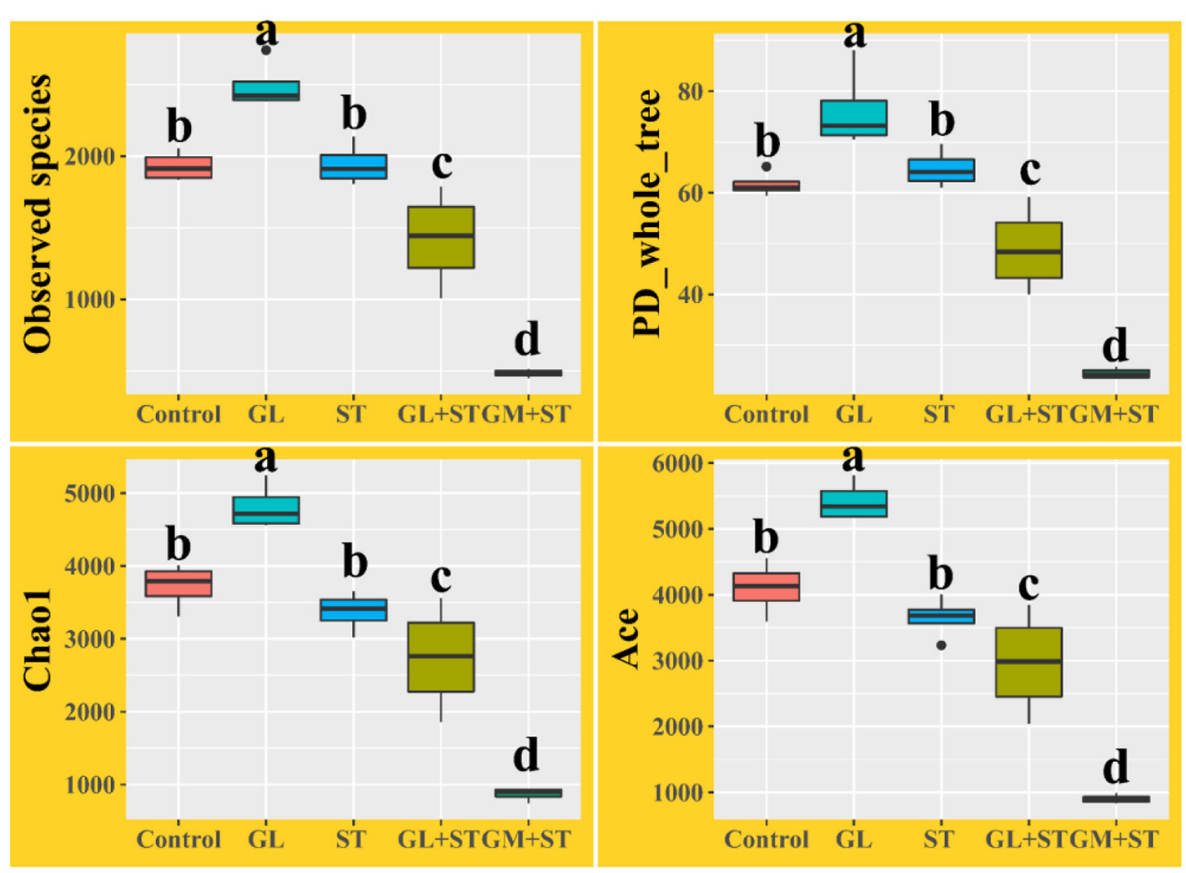

FIGURE 6 | Alpha diversity analysis of intestinal microbiota in different groups ( $n=4$ /group). Different lowercase letters indicate a significant difference $(P<0.05)$.

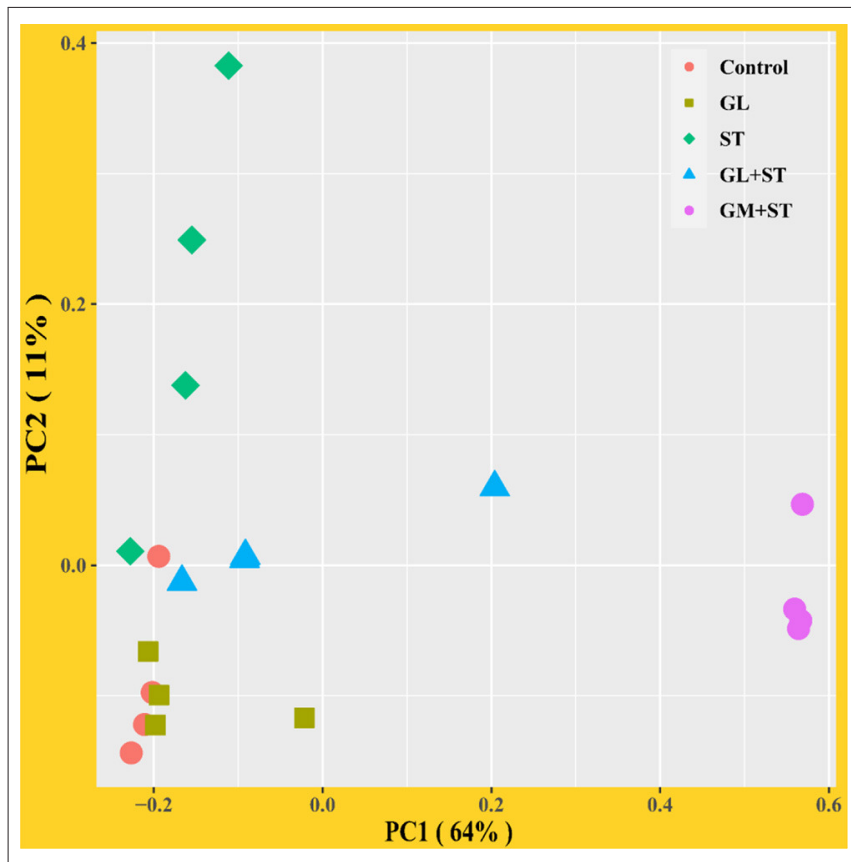

FIGURE 7 | Principal coordinates analysis (PCoA) of microbial communities among groups based on Bray-Curtis distance ( $n=4 /$ group).

group, Ruminiclostridium 9, and Lachnospiraceae UCG001. ST infection significantly $(P<0.05)$ increased the relative abundance of Alphaproteobacteria, Verrucomicrobiales,
TABLE 2 | ANOSIM, PERMANOVA, and MRPP analysis of microbial diversity among different treatments $(n=4 /$ group).

\begin{tabular}{lcccccc}
\hline & \multicolumn{2}{c}{ ANOSIM } & \multicolumn{2}{c}{ ADONIS } & \multicolumn{2}{c}{ MRPP } \\
\cline { 2 - 7 } & $\boldsymbol{R}$ & $\boldsymbol{P}$ & $\boldsymbol{R}^{\mathbf{2}}$ & $\boldsymbol{P}$ & $\boldsymbol{A}$ & $\boldsymbol{P}$ \\
\hline Treatment & 0.653 & 0.001 & 0.765 & 0.001 & 0.451 & 0.001 \\
Control vs. GL & 0.000 & 0.586 & 0.202 & 0.129 & 0.034 & 0.181 \\
Control vs. ST & 0.688 & 0.028 & 0.457 & 0.045 & 0.204 & 0.034 \\
ST vs. GL + ST & 0.313 & 0.071 & 0.327 & 0.023 & 0.111 & 0.029 \\
ST vs. GM + ST & 1.000 & 0.032 & 0.848 & 0.043 & 0.577 & 0.023 \\
GL + ST vs. GM + ST & 1.000 & 0.028 & 0.722 & 0.024 & 0.450 & 0.021 \\
\hline
\end{tabular}

DTU014, Prevotellaceae NK3B31 group, and Ruminococcaceae UCG-013, whereas, it decreased $(P<0.05)$ the relative abundance of Desulfovibrionaceae, Rikenellaceae, ASF356, Rikenella, Ruminococcaceae UCG-009, Lachnospiraceae UCG010, Alistipes, Odoribacter, and Muribaculum. Moreover, GL or GM pretreatments significantly $(P<0.05)$ decreased STinduced relative abundance of Verrucomicrobiales, DTU014, Prevotellaceae NK3B31 group, and Ruminococcaceae UCG-013. The relative abundances of Rikenellaceae and Alistipes were significantly $(P<0.05)$ increased in the GM + ST group compared with those in the ST group.

To determine the co-occurrence patterns of microbes in the different groups, five networks were constructed based on the genus and species levels (Figure 9 and Table 3). Network analysis showed that the values of average degree and graph density in the ST group were higher than those of the other groups 

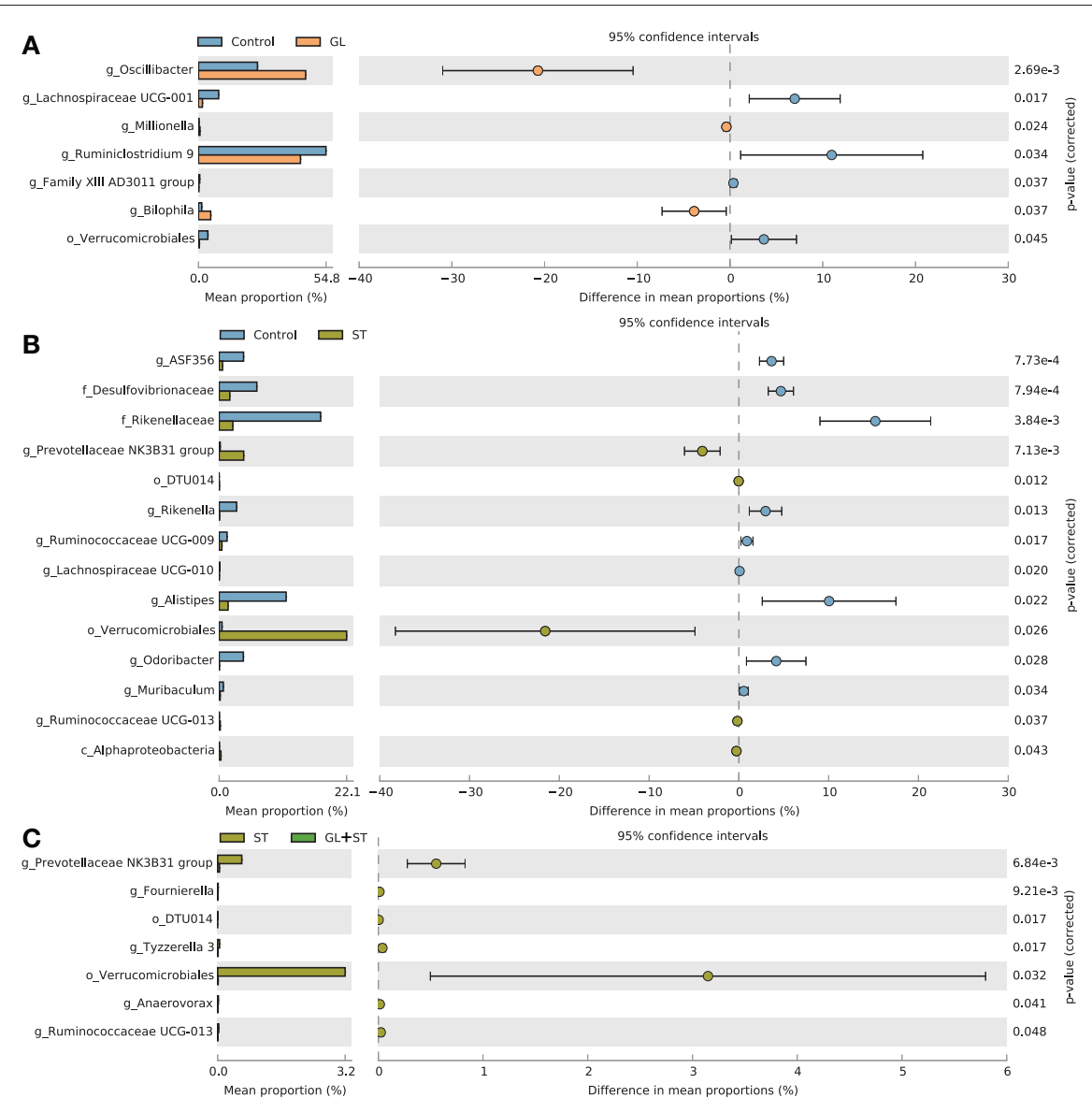

D
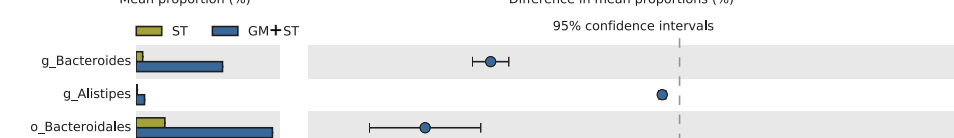

$9.80 \mathrm{e}-7$

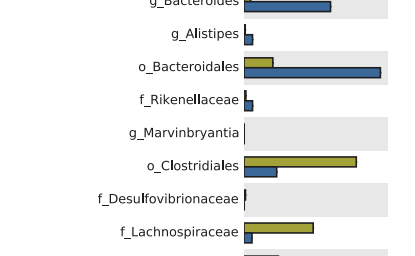

g_Lachnospiraceae NK4A136 group g_Prevotellaceae NK3B31 group $『$

f_Peptococcaceae |

f_Ruminococcaceae $\square$

g_Flavonifractor |

g_Desulfovibrio|

g_Tyzzerella 3 |

o_DTU014 |

f_Prevotellaceae P

g_Candidatus Saccharimonas |

g_Ruminiclostridium ]

g_Anaerovorax |

g_Helicobacter 『

o_Verrucomicrobiales $\boxminus$

g_Ruminococcaceae UCG-013 |

g_Intestinimonas |

f_Muribaculaceae —
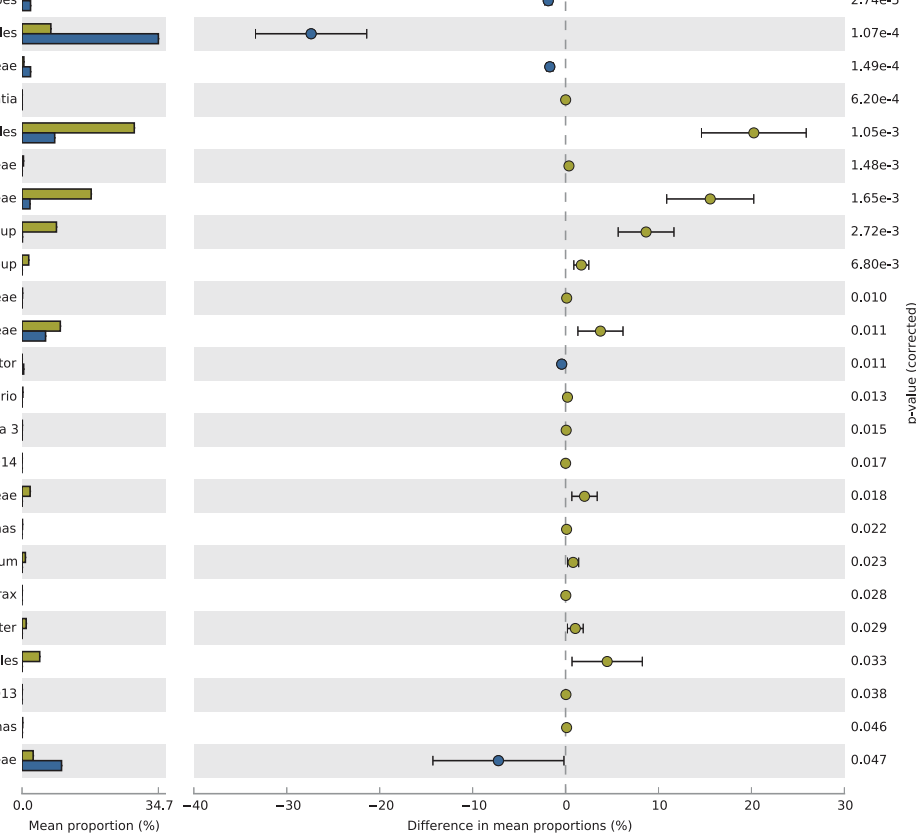

FIGURE 8 | Comparison of the intestinal bacteria among different treatments. (A) Control vs. GL, (B) Control vs. ST, (C) ST vs. GL + ST, (D) Control vs. GM + ST. Confidence Interval was set at $95 \%$. 


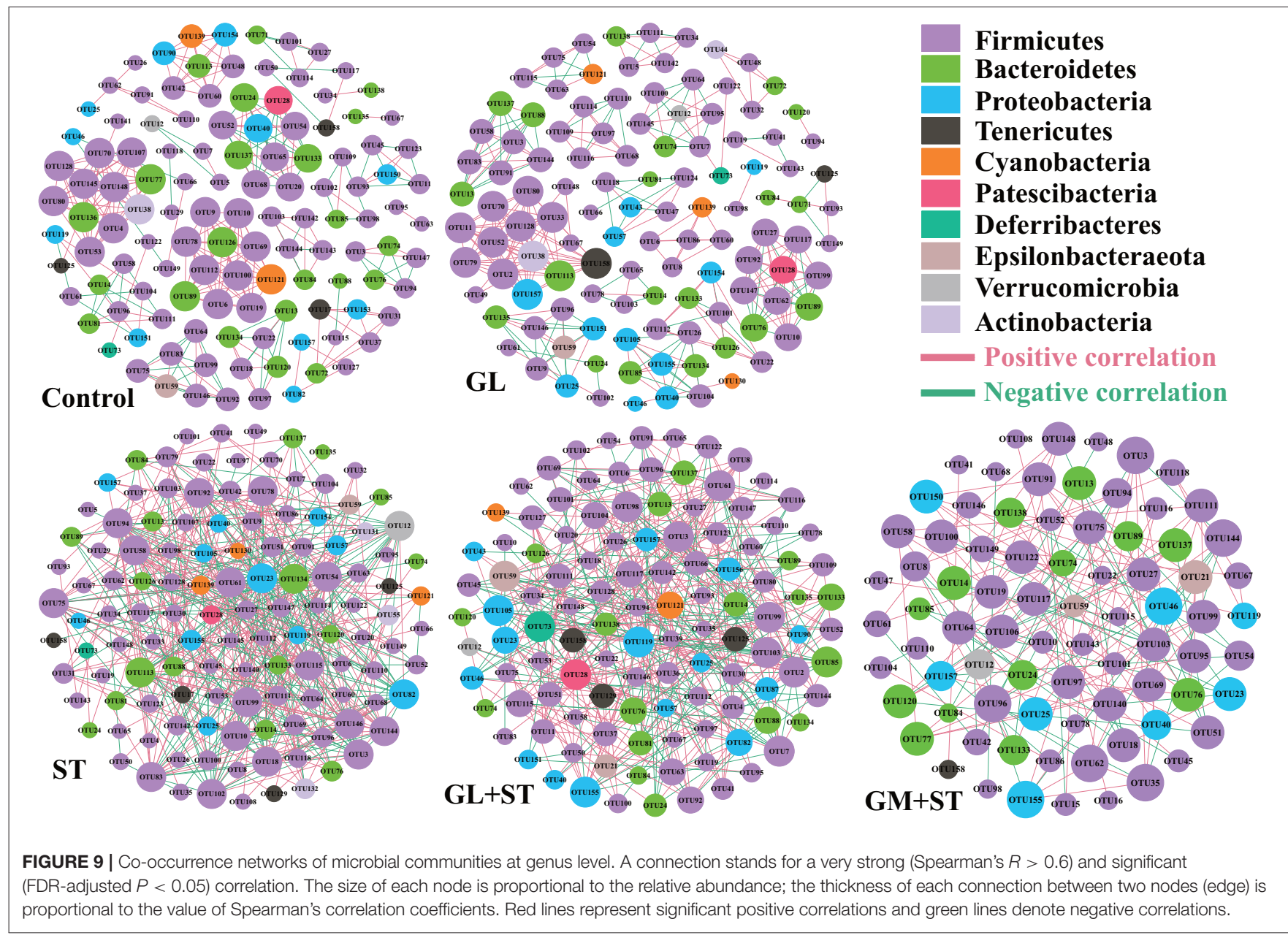

TABLE 3 | Topological properties of co-occurrence network.

\begin{tabular}{lccccc}
\hline & Control & GL & ST & GL + ST & GM + ST \\
\hline Nodes & 120 & 115 & 125 & 114 & 79 \\
Edges & 303 & 298 & 428 & 366 & 146 \\
Average degree (AD) & 5.05 & 5.183 & 6.848 & 6.421 & 3.696 \\
Graph density (GD) & 0.042 & 0.045 & 0.055 & 0.054 & 0.047 \\
Modularity (MD) & 0.893 & 0.896 & 0.735 & 0.877 & 0.912 \\
Positive correlation & $71.95 \%$ & $67.11 \%$ & $56.54 \%$ & $62.57 \%$ & $64.38 \%$ \\
Negative correlation & $28.05 \%$ & $32.89 \%$ & $43.46 \%$ & $37.43 \%$ & $35.62 \%$ \\
\hline
\end{tabular}

(Control, GL, GL + ST, and GM + ST). The modularity values of the co-occurrence networks in all groups were higher than 0.4 . The modularity value in the ST group was lower than those in the other groups (Control, GL, GL + ST, and GM + ST). Additionally, negative correlation of the network in the ST group was more than those in the other groups (Control, GL, GL + ST, and $\mathrm{GM}+\mathrm{ST}$ ).

Pearson's correlation coefficient was calculated to reveal correlations between the phenotypic variables and the relative abundance of microbial communities (phylum level) (Figure 10). Correlation analysis showed that the relative abundances of Verrucomicrobia, Cyanobacteria, Tenericutes, and Actinobacteria were negatively correlated with the final body weight $(P<$ 0.05 or $P<0.01)$. The relative abundances of Verrucomicrobia and Cyanobacteria were positively correlated with the spleen index, caspase- 1 activity, and apoptosis index $(P<0.05$ or $P<$ 0.01 ), while the relative abundance of Tenericutes was positively correlated with caspase-1 activity $(P<0.01)$ and apoptosis index $(P<0.05)$.

\section{DISCUSSION}

Salmonellosis, caused by food-borne Salmonella, is largely associated with tissue injury and intestinal dysbiosis, which accounts for approximately 109.9 million cases and 264,300 deaths globally per year (34). With the strict prohibition of AGP, there is an increasing interest in exploring dietary natural extract products to reduce or prevent against zoonotic enteric pathogen infection and food-borne contamination in industrial livestock production. Previous studies found that 


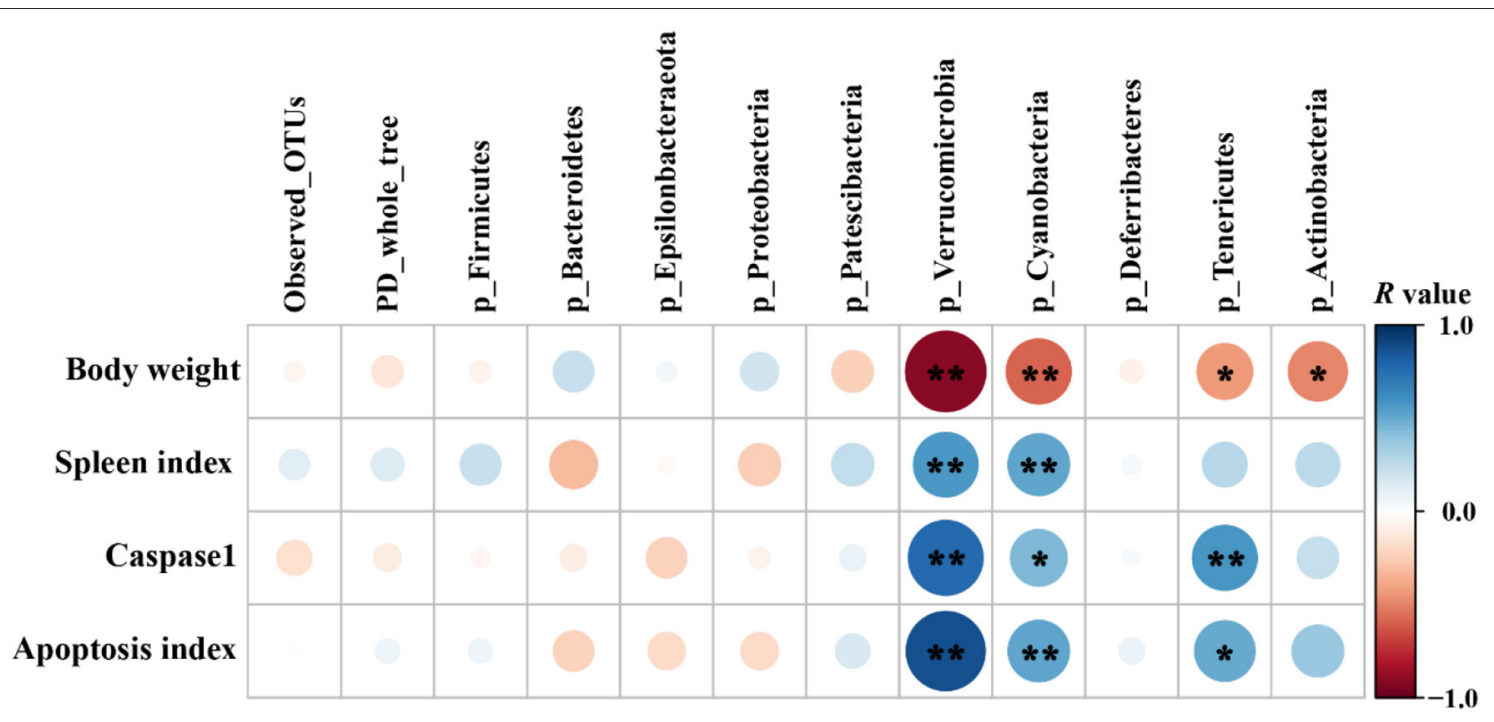

FIGURE 10 | Pearson's correlation analysis between phenotypic variables and the relative abundance of microbial communities (phylum level, $n=4 /$ group). The color and dot size represent the correlation coefficient within rows. ${ }^{*} P<0.05$; ${ }^{* *} P<0.01$.

GL, an extract from licorice root, could ameliorate HFDmediated intestinal dysbiosis (20) and Streptococcus aureusinduced acute lung injury (25) in murine models. Our previous study found that GL could effectively ameliorate the body weight loss of mice infected with $S$. Typhimurium infection (30), which may be related to the beneficial effects of GL in reducing the inflammation and tissue damage associated with $S$. Typhimurium infection. Salmonella Typhimurium may migrate to the liver and spleen via the blood circulatory system due to the damage of the intestinal barrier to cause the hepatosplenomegaly, which is characterized by cell apoptosis and inflammatory infiltration $(35,36)$. In the present study, we found that GL pretreatment significantly prevented $S$. Typhimuriuminduced jejunal injury indicated by the higher villous height and splenomegaly indicated by the decreased the spleen index of mice. Additionally, liver injury induced by $S$. Typhimurium infection was also relieved by GL or GM pretreatments as evidenced by the decreased hepatocytic apoptosis and the reduced caspase- 1 enzyme activity. GL pretreatment significantly inhibited $S$. Typhimurium colonization in ileum and colon and translocation to spleen and liver (30). In addition, GL enhanced the phagocytosis and bactericidal capacity of BMDM by inducing the JNK/NF-??B signaling pathway-mediated polarization of M1 macrophages that participate in polarized Th1 responses and act as the primary line of defense against intracellular pathogens $(18,37)$. These results indicate that the attenuated injury of tissues in the GL + ST group may be related to the inhibition of colonization and invasion of $S$. Typhimurium (30), polarization of M1 macrophages (18), improved inflammation response, and intestinal dysbacteriosis.

Inflammatory responses induced by pathogenic infection is one of the most common symptoms of splenomegaly and hepatomegaly (38). The expression of pro-inflammatory cytokines (such as IFN- $\gamma$, IL-6, IL-12, and TNF- $\alpha$ ) is necessary for the initiation of the innate immune response for pathogen clearance (39). However, excessive pro-inflammatory responses could be extremely harmful to the host (40). Thus, excessive pro-inflammatory responses are tightly balanced by antiinflammatory cytokines (such as IL-10 and TGF- $\beta$ ) and associated negative feedback loops (41). The ameliorated tissue injury of $S$. Typhimurium-infected mice in GL- or GMpretreated group mentioned above may be due to the attenuated inflammatory response. Indeed, compared with the uninfected group, S. Typhimurium infection significantly induced inflammatory responses as evidenced by the upregulation of pro-inflammatory cytokines (IFN- $\gamma$, IL-6, IL-12, and TNF- $\alpha$ ) and anti-inflammatory cytokine IL-10 in spleen and liver of mice. Interestingly, GL pretreatment effectively decreased the upregulation of pro-inflammatory cytokines (IFN- $\gamma$, TNF- $\alpha$, and IL-6) induced by ST infection in spleen and liver, and increased secretion of anti-inflammatory cytokine IL-10 in spleen. The results indicate that GL effectively alleviates $S$. Typhimurium-induced inflammatory response by exerting an anti-inflammatory activity.

Intestinal microbes play an important role in maintaining gastrointestinal homeostasis. The intestinal beneficial and commensal microbiota protect host against Salmonella colonization via competition of adhesion site and nutrition and secretion of metabolite (SCFAs, indole, and bacteriocin) $(42,43)$. It is reported that $S$. Typhimurium infection is tightly accompanied by intestinal dysbiosis $(44,45)$. Salmonella outcompetes the resident microbes by inducing the host intestinal immune system to secrete reactive oxygen species (ROS) and antimicrobial peptides that are non-specific harmful to the beneficial and commensal microbes (43). In this study, we observed that Salmonella infection led to dysbiosis by altering microbial beta diversity and composition. Salmonella Typhimurium infection significantly increased the abundances 
of Alphaproteobacteria, Verrucomicrobiales, DTU014, Prevotellaceae NK3B31 group, and Ruminococcaceae UCG-013, whereas, it decreased the abundances of Desulfovibrionaceae, Rikenellaceae, ASF356, Rikenella, Ruminococcaceae UCG009, Lachnospiraceae UCG-010, Alistipes, Odoribacter, and Muribaculum. Knowledge about the altered bacteria caused by $S$. Typhimurium infection remains unclear and needs further investigation. Oral administration of GL significantly increased microbial alpha diversity and altered microbial composition. GL significantly increased the relative abundances of Oscillibacter, Millionella, and Bilophila, whereas, it decreased the relative abundances of Verrucomicrobiales, Family XIII AD3011 group, Ruminiclostridium 9, and Lachnospiraceae UCG-001. Oscillibacter was proposed to exert an anti-inflammatory effect (46). Administration of probiotics increased cecal Millionella abundance of mice (47). Bilophila, a sulfate-reducing bacterium, is associated with animal-based and fat-enriched diets (48). The intestinal dysbiosis caused by Salmonella infection can be reshaped by dietary or antibiotic interventions $(31,49)$. In the present study, GL or GM pretreatments significantly reshaped the intestinal microbes by reducing microbial alpha diversity, modulating beta diversity, and microbial compositions. The results indicate that the altered microbial diversities and compositions induced by GL pretreatment may be related to the attenuated injury of tissues and pro-inflammatory responses induced by $S$. Typhimurium infection, although, more direct evidences are needed.

Co-occurrence pattern analysis was performed to investigate the microbial interactions, and we found that the values of average degree and graph density of microbial network in the $S$. Typhimurium infected group were higher than those of the other groups (Control, GL, GL + ST, and GM + $\mathrm{ST})$, suggesting that $S$. Typhimurium infection increased the connection among the microbes. All the modularity values of the co-occurrence networks were higher than 0.4, suggesting that these networks had a modular structure (50). The microbial network in the $S$. Typhimurium-infected group was not easier to form a "small world" as evidenced by lower modularity value than those of the other groups (33). Additionally, negative correlation of the microbial networks in the other groups was less than that of the $S$. Typhimurium-infected group, which could be interpreted as a reduction in competitive relationships within intestinal microbes (51). Finally, the correlations between phenotypic variables and microbial communities (alpha diversity and phylum level) were further investigated. The final body weight was obtained from our previous study (30) and reanalyzed with the relative abundance of bacterial phyla. Pearson's correlation analysis showed that the final body weight of mice was negatively correlated with cecal Verrucomicrobia, Cyanobacteria, Tenericutes, and Actinobacteria. Verrucomicrobia and Cyanobacteria were positively correlated with the spleen index, caspase-1 activity, and apoptosis index. Tenericutes was positively correlated with caspase- 1 activity and apoptosis index. The above results indicate that the relieved negative effect caused by $S$. Typhimurium infection may be related to the altered microbial diversities and compositions induced by GL pretreatment.

\section{CONCLUSION}

Collectively, the present study demonstrates that GL exerts the anti-inflammatory and tissue-protective properties to attenuate ST infection, as indicated by alleviating ST-induced splenomegaly, hepatocytic apoptosis, injury of jejunum and liver, and inflammatory response of liver and spleen in mice. Moreover, we found that GL modulates ST-induced intestinal dysbacteriosis. These findings might give a new perspective into the function of GL in regulating the host immune and intestinal microbiota to defense against pathogen infection. However, further studies about the role of the intestinal microbiota in the progress of GL-mediated anti-Salmonella infection are needed.

\section{ELISA KIT INFORMATION}

According to the manufacturer's instructions (eBioscience, San Diego, CA), the detailed information about the used ELISA kits is IFN- $\gamma$ (Assay range: $15.6-1,000 \mathrm{pg} / \mathrm{ml}$; Analytical sensitivity: $1.7 \mathrm{pg} / \mathrm{ml}$; Intra- and Inter-assay CV: $<6.8$ and $<7.4 \%$ ), IL-12p70 (Assay range: $15.6-1,000 \mathrm{pg} / \mathrm{ml}$; Analytical sensitivity: 10.0 $\mathrm{pg} / \mathrm{ml}$; Intra- and Inter-assay CV: 8.3 and 11.0\%), TNF- $\alpha$ (Assay range: $31.3-2000 \mathrm{pg} / \mathrm{ml}$; Analytical sensitivity: $3.7 \mathrm{pg} / \mathrm{ml}$; Intra- and Inter-assay CV: 6.5 and 5.7\%), IL-6 (Assay range: 31.3-2,000 pg/ml; Analytical sensitivity: $6.5 \mathrm{pg} / \mathrm{ml}$; Intra- and Inter-assay CV: 5 and 8.9\%), and IL-10 (Assay range: 39.1-2,500 pg/ml; Analytical sensitivity: $5.28 \mathrm{pg} / \mathrm{ml}$; Intra- and Inter-assay CV: 6.7 and 10.1\%).

\section{DATA AVAILABILITY STATEMENT}

Raw sequences have been deposited in the Genome Sequence Archive (GSA) of the BIG Data Center (https://bigd.big.ac.cn/gsa/) under accession number PRJCA004208/CRA003756.

\section{ETHICS STATEMENT}

All animal experiments were conducted according to the guidelines and approval of the Institutional Animal Care and Use Committee of Zhejiang University (Permission number: ZJU20160416).

\section{AUTHOR CONTRIBUTIONS}

YM: conceptualization, supervision, and project administration. BW, XY, and PZ: writing-original draft preparation and writing-review and editing. BW, YZ, and PZ: investigation, methodology, and visualization. All authors have read and agreed to the published version of the manuscript.

\section{FUNDING}

This research was funded by the Education Foundation of Da Bei Nong Group. 


\section{REFERENCES}

1. LaRock DL, Chaudhary A, Miller SI. Salmonellae interactions with host processes. Nat Rev Microbiol. (2015) 13:191-205. doi: 10.1038/nrmicro 3420

2. Haraga A, Ohlson MB, Miller SI. Salmonellae interplay with host cells. Nat Rev Microbiol. (2008) 6:53-66. doi: 10.1038/nrmicro1788

3. Liu JS, Gu ZN, Lu WW, Hu DG, Zhao X, Huang HX, et al. Multiple mechanisms applied by Lactobacillus pentosus AT6 to mute the lethal effects of Salmonella in a mouse model. Food Funct. (2018) 9:278795. doi: 10.1039/C7FO01858D

4. Cossart P, Sansonetti PJ. Bacterial invasion: the paradigms of enteroinvasive pathogens. Science. (2004) 304:242-8. doi: 10.1126/science.1090124

5. Als D, Radhakrishnan A, Arora P, Gaffey MF, Campisi S, Velummailum R, et al. Global trends in typhoidal Salmonellosis: a systematic review. Am J Trop Med Hyg. (2018) 99:10-9. doi: 10.4269/ajtmh.18-0034

6. Kim HB, Isaacson RE. Salmonella in swine: microbiota interactions. Ann Rev Anim Biosci. (2017) 5:43-63. doi: 10.1146/annurev-animal-022516-022834

7. Cox NA, Cason JA, Richardson LJ. Minimization of Salmonella contamination on raw poultry. Annu Rev Food Sci T. (2011) 2:75-95. doi: 10.1146/annurev-food-022510-133715

8. Finstad S, O’Bryan CA, Marcy JA, Crandall PG, Ricke SC. Salmonella and broiler processing in the United States: relationship to foodborne Salmonellosis. Food Res Int. (2012) 45:789-94. doi: 10.1016/j.foodres.2011.03.057

9. Eng SK, Pusparajah P, Ab Mutalib NS, Ser HL, Chan KG, Lee LH. Salmonella: a review on pathogenesis, epidemiology and antibiotic resistance. Front Life Sci. (2015) 8:284-93. doi: 10.1080/21553769.2015. 1051243

10. Broom LJ. The sub-inhibitory theory for antibiotic growth promoters. Poultry Sci. (2017) 96:3104-8. doi: 10.3382/ps/pex114

11. Teillant A, Brower CH, Laxminarayan R. Economics of antibiotic growth promoters in livestock. Annu Rev Resour Econ. (2015) 7:349-74. doi: 10.1146/annurev-resource-100814-125015

12. China. Announcement of the Ministry of Agriculture and Rural People's Republic of China No. 194. (2019). Available online at: http://www.moa. gov.cn/nybgb/2017/dqq/201801/t20180103_6133925.htm (accessed July 20, 2017).

13. Tellez G, Latorre JD. Editorial: alternatives to antimicrobial growth promoters and their impact in gut microbiota, health and disease. Front Vet Sci. (2017) 4:196. doi: 10.3389/fvets.2017.00196

14. Marshall BM, Levy SB. Food animals and antimicrobials: impacts on human health. Clin Microbiol Rev. (2011) 24:718-33. doi: 10.1128/CMR.00002-11

15. Buntyn JO, Schmidt TB, Nisbet DJ, Callaway TR. The role of direct-fed microbials in conventional livestock production. Annu Rev Anim Biosci. (2016) 4:335-55. doi: 10.1146/annurev-animal-022114-111123

16. FDA. CFR-Code of Federal Regulations Title 21. Washington, DC: US Food Drug Administration (2017).

17. Cheng GY, Hao HH, Xie SY, Wang X, Dai MH, Huang LL, et al. Antibiotic alternatives: the substitution of antibiotics in animal husbandry? Front Microbiol. (2014) 5:217. doi: 10.3389/fmicb.2014.00217

18. Mao YL, Wang BK, Xu X, Du W, Li WF, Wang YM. Glycyrrhizic acid promotes M1 macrophage polarization in murine bone marrow-derived macrophages associated with the activation of JNK NF-kappa B. Mediat Inflamm. (2015) 2015:372931. doi: 10.1155/2015/372931

19. Matsui S, Matsumoto H, Sonoda Y, Ando K, Aizu-Yokota E, Sato $\mathrm{T}$, et al. Glycyrrhizin and related compounds down-regulate production of inflammatory chemokines IL-8 and eotaxin 1 in a human lung fibroblast cell line. Int Immunopharmacol. (2004) 4:1633-44. doi: 10.1016/j.intimp.2004.07.023

20. Qiu M, Huang KQ, Liu YZ, Yang YQ, Tang HL, Liu XX, et al. Modulation of intestinal microbiota by glycyrrhizic acid prevents high-fat diet-enhanced pre-metastatic niche formation and metastasis. Mucosal Immunol. (2019) 12:945-57. doi: 10.1038/s41385-019-0144-6

21. Cinatl J, Morgenstern B, Bauer G, Chandra P, Rabenau H, Doerr HW. Glycyrrhizin, an active component of liquorice roots, and replication of SARS-associated coronavirus. Lancet. (2003) 361:2045-6. doi: 10.1016/S0140-6736(03)13615-X
22. Wang Y, Chen Q, Shi CX, Jiao FZ, Gong ZJ. Mechanism of glycyrrhizin on ferroptosis during acute liver failure by inhibiting oxidative stress. Mol Med Rep. (2019) 20:4081-90. doi: 10.3892/mmr.2019.10660

23. Bordbar N, Karimi MH, Amirghofran Z. The effect of glycyrrhizin on maturation and $\mathrm{T}$ cell stimulating activity of dendritic cells. Cell Immunol. (2012) 280:44-9. doi: 10.1016/j.cellimm.2012.11.013

24. Itoh K. Augmentation of NK Activity by Several Anti-Inflammatory Agents. 3rd ed. Int Cong Series. Excerpta Medica, Amsterdam (1983). p. 460-4.

25. Yao L, Sun TL. Glycyrrhizin administration ameliorates Streptococcus aureus-induced acute lung injury. Int Immunopharmacol. (2019) 70:50411. doi: 10.1016/j.intimp.2019.02.046

26. Carruthers NJ, McClellan SA, Somayajulu M, Pitchaikannu A, Bessert D, Peng XD, et al. Effects of glycyrrhizin on multi-drug resistant pseudomonas aeruginosa. Pathogens. (2020) 9:776. doi: 10.3390/pathogens 9090766

27. Rohinishree YS, Negi PS. Effect of licorice extract on cell viability, biofilm formation and exotoxin production by Staphylococcus aureus. J Food Sci Tech Mys. (2016) 53:1092-100. doi: 10.1007/s13197-015-2131-6

28. Naser M, Shahab G, Mahmood H. Drinking water supplementation of licorice (Glycyrrhiza glabra L. root) extract as an alternative to in-feed antibiotic growth promoter in broiler chickens. GSC Biol Pharm Sci. (2017) 1:208. doi: 10.30574/gscbps.2017.1.3.0039

29. Ibrahim D, Sewid AH, Arisha AH, abd El-fattah AH, Abdelaziz AM, Al-Jabr $\mathrm{OA}$, et al. Influence of glycyrrhiza glabra extract on growth, gene expression of gut integrity, and campylobacter jejuni colonization in broiler chickens. Front Vet Sci. (2020) 7:612063. doi: 10.3389/fvets.2020.612063

30. Xu XG, Gong L, Wang BK, Wu YP, Wang Y, Mei XQ, et al. Glycyrrhizin attenuates Salmonella enterica serovar Typhimurium infection: new insights into its protective mechanism. Front Immunol. (2018) 9:2321. doi: 10.3389/fimmu.2018.02321

31. Fu A, Mo Q, Wu Y, Wang B, Liu R, Tang L, et al. Protective effect of Bacillus amyloliquefaciens against Salmonella via polarizing macrophages to M1 phenotype directly and to M2 depended on microbiota. Food Funct. (2019) 10:7653-66. doi: 10.1039/C9FO01651A

32. Jiao S, Chen WM, Wang JM, Zhang L, Yang F, Lin YB, et al. Plant growth and oil contamination alter the diversity and composition of bacterial communities in agricultural soils across China. Land Degrad Dev. (2018) 29:1660-71. doi: 10.1002/ldr.2932

33. Jiao S, Liu ZS, Lin YB, Yang J, Chen WM, Wei GH. Bacterial communities in oil contaminated soils: biogeography and co-occurrence patterns. Soil Biol Biochem. (2016) 98:64-73. doi: 10.1016/j.soilbio.2016.04.005

34. Stanaway JD, Parisi A, Sarkar K, Blacker BF, Reiner RC, Hay SI, et al. The global burden of non-typhoidal Salmonella invasive disease: a systematic analysis for the Global Burden of Disease Study 2017. Lancet Infect Dis. (2019) 19:1312-24. doi: 10.1016/S1473-3099(19)30418-9

35. Wu JH, Hu YJ, Du CM, Piao JH, Yang LC, Yang XG. The effect of recombinant human lactoferrin from the milk of transgenic cows on Salmonella enterica serovar Typhimurium infection in mice. Food Funct. (2016) 7:308-14. doi: 10.1039/C5FO00817D

36. Mathur R, Oh H, Zhang DK, Park SG, Seo J, Koblansky A, et al. A mouse model of Salmonella typhi infection. Cell. (2012) 151:590602. doi: 10.1016/j.cell.2012.08.042

37. Wang BK, Wu YP, Liu RR, Xu H, Mei XQ, Shang QQ, et al. Lactobacillus rhamnosus GG promotes M1 polarization in murine bone marrow-derived macrophages by activating TLR2/MyD88/MAPK signaling pathway. Anim Sci J. (2020) 91:e13439. doi: 10.1111/asj.13439

38. Ohshio G, Furukawa F, Fujiwara H, Hamashima Y. Hepatomegaly and splenomegaly in Kawasaki disease. Pediatr Pathol. (1985) 4:25764. doi: 10.3109/15513818509026899

39. Lee MS, Min YJ. Signaling pathways downstream of pattern-recognition receptors and their cross talk. Annu Rev Biochem. (2007) 76:44780. doi: 10.1146/annurev.biochem.76.060605.122847

40. Kawasaki T, Kawai T. Toll-like receptor signaling pathways. Front Immunol. (2014) 5:461. doi: 10.3389/fimmu.2014.00461

41. Wang B, Hussain A, Zhou Y, Zeng Z, Wang Q, Zou P, et al. Saccharomyces boulardii attenuates inflammatory response induced by Clostridium perfringens via TLR4/TLR15-MyD8 pathway in HD11 avian macrophages. Poult Sci. (2020) 99:5356-65. doi: 10.1016/j.psj.2020. 07.045 
42. Caballero S, Pamer EG. Microbiota-mediated inflammation and antimicrobial defense in the intestine. Annu Rev Immunol. (2015) 33:227-56. doi: 10.1146/annurev-immunol-032713-120238

43. Gart EV, Suchodolski JS, Welsh TH Jr, Alaniz RC, Randel RD, et al. Salmonella Typhimurium and multidirectional communication in the gut. Front Microbiol. (2016) 7:1827. doi: 10.3389/fmicb.2016.01827

44. Gillis CC, Hughes ER, Spiga L, Winter MG, Zhu W, Furtado de Carvalho T, et al. Dysbiosis-associated change in host metabolism generates lactate to support Salmonella growth. Cell Host Microbe. (2018) 23:54-64 e6. doi: 10.1016/j.chom.2017.11.006

45. Khan S, Chousalkar KK. Salmonella Typhimurium infection disrupts but continuous feeding of Bacillus based probiotic restores gut microbiota in infected hens. J Anim Sci Biotechnol. (2020) 11:29. doi: 10.1186/s40104-020-0433-7

46. Li J, Sung CY, Lee N, Ni Y, Pihlajamaki J, Panagiotou G, et al. Probiotics modulated gut microbiota suppresses hepatocellular carcinoma growth in mice. Proc Natl Acad Sci U S A. (2016) 113:E1306-15. doi: 10.1073/pnas.1518189113

47. Wang F, Xu T, Zhang Y, Zheng T, He Y, He F, et al. Long-term combined administration of Bifidobacterium bifidum TMC3115 and Lactobacillus plantarum 45 alleviates spatial memory impairment and gut dysbiosis in APP/PS1 mice. FEMS Microbiol Lett. (2020) 367:fnaa048. doi: 10.1093/femsle/fnaa048

48. Natividad JM, Lamas B, Pham HP, Michel ML, Rainteau D, Bridonneau $\mathrm{C}$, et al. Bilophila wadsworthia aggravates high fat diet induced metabolic dysfunctions in mice. Nat Commun. (2018) 9:2802. doi: 10.1038/s41467-018-05249-7

49. Wang X, Kong X, Qin Y, Zhu X, Qu D, Han J. Milk phospholipid supplementation mediates colonization resistance of mice against Salmonella infection in association with modification of gut microbiota. Food Funct. (2020) 11:6078-90. doi: 10.1039/D0FO00883D

50. Newman MEJ. Modularity and community structure in networks. P Natl Acad Sci USA. (2006) 103:8577-82. doi: 10.1073/pnas.0601602103

51. Fan KK, Weisenhorn P, Gilbert JA, Shi Y, Bai Y, Chu HY. Soil $\mathrm{pH}$ correlates with the co-occurrence and assemblage process of diazotrophic communities in rhizosphere and bulk soils of wheat fields. Soil Biol Biochem. (2018) 121:185-92. doi: 10.1016/j.soilbio.2018. 03.017

Conflict of Interest: The authors declare that the research was conducted in the absence of any commercial or financial relationships that could be construed as a potential conflict of interest.

Copyright (c) 2021 Wang, Ye, Zhou, Zhao and Mao. This is an open-access article distributed under the terms of the Creative Commons Attribution License (CC BY). The use, distribution or reproduction in other forums is permitted, provided the original author(s) and the copyright owner(s) are credited and that the original publication in this journal is cited, in accordance with accepted academic practice. No use, distribution or reproduction is permitted which does not comply with these terms. 